\title{
Outcome After Therapeutic Lymph Node Dissection in Patients with Unknown Primary Melanoma Site
}

\author{
Sebastiaan P. Prens, MSc ${ }^{1}$, Augustinus P. T. van der Ploeg, MSc ${ }^{1}$, Alexander C. J. van Akkooi, MD, PhD ${ }^{1}$, \\ Cornelis A. G. M. van Montfort, $\mathrm{PhD}^{1}$, Albertus N. van Geel, MD, $\mathbf{P h D}^{1}$, Johannes H. W. de Wilt, MD, $\mathbf{P h D}^{2}$, \\ Alexander M. M. Eggermont, MD, $\mathbf{P h D}^{1,3}$, and Cornelis Verhoef, $\mathrm{MD}, \mathrm{PhD}^{1}$ \\ ${ }^{1}$ Erasmus University Medical Center, Daniel den Hoed Cancer Center, Rotterdam, The Netherlands; ${ }^{2}$ Radboud University \\ Nijmegen Medical Center, Nijmegen, The Netherlands; ${ }^{3}$ Institut de Cancérologie Gustave Roussy, Villejuif, France
}

\begin{abstract}
Purpose. The aim of this study was to evaluate the incidence and outcome of melanoma of unknown primary site (MUP) after therapeutic lymph node dissection (TLND) of palpable nodal melanoma metastases. Disease-free (DFS) and overall survival (OS) time of MUP patients were analyzed and compared to patients undergoing a TLND for known primary melanomas (MKP).

Methods. This single institution retrospective study analyzed 342 consecutive patients who were treated with 415 TLNDs for palpable nodal disease from 1982 to 2009. Univariate and multivariate analyses included: MUP versus MKP, gender, Breslow thickness, ulceration of primary tumor, site of primary tumor, site of dissection, extracapsular extension, number of collected nodes, number of positive nodes and the node positive ratio.

Results. A total of 47 MUP were identified in 342 patients (13.7\%). In univariate analysis, a trend was seen toward better survival for MUP patients compared to MKP patients having 5-year OS rates of $40 \%$ and $27 \%$, respectively $(P=0.06)$. Multivariate analysis for $\mathrm{OS}$ showed two highly significant factors associated with worse prognosis: extracapsular extension and N3 status (both $P<0.001$ ). Two factors were associated with a significant better prognosis: MUP $(P=0.03)$ and a neck dissection $(P=0.04)$.
\end{abstract}

Conclusions. Patients with MUP showed a statistically significant better OS compared to patients with melanoma metastases from known primary tumors. Presence of

(C) The Author(s) 2011. This article is published with open access at Springerlink.com

First Received: 23 March 2011;

Published Online: 25 May 2011

C. Verhoef, MD, PhD

e-mail: c.verhoef@erasmusmc.nl extracapsular extension and an increased number of positive nodes are statistically significantly negative prognostic factors for OS. The absence of a primary melanoma in stage III melanoma patients does not preclude surgery.

First presentation of palpable nodal disease in melanoma patients still occurs in spite of early recognition programs leading to a decrease in Breslow thickness. ${ }^{1}$ Approximately $4 \%$ to $9 \%$ of all patients presenting with melanoma are diagnosed with palpable nodal disease, i.e., stage III disease. ${ }^{2,3}$ Patients with clinically detected and histologically confirmed nodal melanoma metastases with no identification of a primary site are diagnosed as patients with melanoma of unknown primary site (MUP). In $8 \%$ to $20 \%$ of all therapeutic lymph node dissections (TLND) for regional metastatic melanoma, no primary tumor can be found. ${ }^{4,5}$ Possible explanations for the absence of a primary tumor are spontaneous regression, unidentified primary melanoma, previous excision of what was considered a benign lesion or a malignant transformation of an ectopic nodal melanocyte. ${ }^{4,6}$ Whether patients with MUP have better or worse prognosis than patients with melanoma of known primary site (MKP) presented with nodal metastases is uncertain. Some studies suggest an improved survival for patients with MUP compared to MKP, whereas others report similar survival or even worse survival for MUP patients. ${ }^{3-5,7}$

The aim of this study was to evaluate the incidence and outcome of MUP patients after TLND for palpable nodal disease compared to patients undergoing a TLND for palpable nodal disease with a known primary tumor. Diseasefree survival (DFS) and overall survival (OS) were analyzed to identify prognostic factors for all patients who underwent TLND. 


\section{PATIENTS AND METHODS}

Patients in this retrospective study were all treated with TLND for palpable nodal disease between 1982 and 2009 at the Erasmus University Medical Center, Daniel den Hoed Cancer Center, Rotterdam, the Netherlands. In 342 melanoma patients, a total of 415 TLNDs were performed. Patients who were treated with a lymph node dissection (LND) because of a positive sentinel node, patients who underwent LND with an isolated limb perfusion and patients who underwent elective LND were excluded.

There was no history of a primary melanoma and no primary tumor could be located in 47 of 342 patients (13.7\%). The control group was formed by 295 patients (86.3\%) with a known primary tumor. The diagnosis of unknown primary melanoma (MUP) consisted of histologically confirmed nodal metastatic melanoma and the absence of a primary tumor, confirmed after thorough examination of the skin and unusual primary sites such as urogenital, nasopharyngeal, or ocular.

All patient, primary and metastatic tumor characteristics were prospectively collected and sorted in a data base. Clinically detectable nodal disease and the absence of visceral metastases was radiographically confirmed by either ultrasound of the lymph node fields and/or the liver, chest $\mathrm{x}$-ray, cerebral magnetic resonance imaging, or computed tomographic scan of the thorax and abdomen.

\section{Surgical Technique}

Four coauthors performed the majority of LNDs assessed for this study (A.N.V.G., J.H.D.W., A.M.M.E. and C.V.). Ilioinguinal dissections or deep groin dissections included dissection of the femoral-inguinal and external iliac nodes up to the common iliac artery (if necessary up to the aorta bifurcation) and dissection of the obturator nodes. Ilioinguinal dissections were performed by using one long vertical incision in the early stage of the study period. Two separate incisions were used in a later stage. Sartorius muscle transposition to cover and protect the femoral vessels was selectively performed when adjuvant radiotherapy was to be expected and/or patient's skin was at risk. An axillary lymphadenectomy comprised dissection for levels I-III. The modified radical neck consists of dissection of level $\mathrm{I}-\mathrm{V}$ with preservation of the spinal accessory muscle, internal jugular vein and sternocleidomastoid muscle. Radical neck dissections were only performed if last mentioned structures were involved in the tumor process. In all patients vacuum drains were placed operatively and removed postoperatively if they produced less than $100 \mathrm{ml}$ in 24 hours. Postoperatively the treatment protocol of all patients consisted of daily wound inspections. No pre-, peri- or postoperative antibiotic prophylaxis was routinely given. Ilioinguinal dissection patients were ordered 3 days of bed rest postoperatively, after which they would be mobilized with the use of a support stocking. Patients received low molecule weight heparin during immobilization. The number and type of complications and duration of hospitalization were recorded.

\section{Adjuvant Therapy}

In the later years of this study, several patients participated in the EORTC 18951, 18952 or 18991 trials. The EORTC 18951 trial found no clinically relevant activity for adding interleukin-2 to a chemoimmunotherapy combination of dacarbazine, cisplatin and interferon alfa (IFN- $\alpha$ ) $2 \mathrm{~b} .^{8}$ The EORTC 18952 trial evaluated the effects of adjuvant therapy with intermediate doses of IFN- $\alpha 2 b$, and did not show a survival benefit for patients in the treatment group. ${ }^{9}$ The EORTC 18991 trial evaluated the role of longterm treatment with pegylated IFN and found a sustained effect on recurrence-free survival. ${ }^{10}$ Seven of 47 (14.9\%) MUP patients and 46 of 295 (15.6\%) of MKP patients participated in these trials.

Adjuvant radiotherapy was considered if narrow resection margins, excessive nodal involvement, i.e., more than three positive lymph nodes, extracapsular extension (ECE), or simultaneous in transit, subcutaneous or skin metastases in the operation area were present.

\section{Statistical Analysis}

All descriptive and survival analyses were performed assessing the 342 patients. The Fisher exact test, chi-square test and Mann-Whitney U test were executed to determine the differences between MUP and MKP patients. DFS was calculated from the first dissection date to the date of first recurrence. OS time was calculated from the dissection date to date of death. Patients without such an event at their last follow-up were censored at that time. Estimates were made according to the Kaplan Meier method and compared with the log rank score. The following factors were evaluated with a univariate Cox regression analysis: age, gender, MUP, location of the affected lymph node basin, the number of tumor positive lymph nodes, node-positive ratio ( $\mathrm{N}$ ratio; total affected lymph nodes/total collected nodes), ECE and adjuvant radiotherapy. The number of positive lymph nodes was defined by the American Joint Committee on Cancer (AJCC) 2009 classification, i.e., N1 (one positive lymph node), N2 (two or three positive lymph nodes) and N3 (more than three positive lymph nodes). Multivariate analysis by Cox's proportional hazards regression model was performed with all variables reaching a significance level of $10 \%$ in the univariate models. A stepwise backward algorithm was used at a level of $5 \%$ 
significance to exclude factors. All statistical analyses were performed by SPSS software (PASW 17.0.2; SPSS, Chicago, IL).

\section{RESULTS}

A total of 342 patients were treated with TLND. A melanoma of unknown primary (MUP) was diagnosed in $47(13.7 \%)$ patients and 295 patients $(86.3 \%)$ had a known primary tumor (MKP). Two or more dissections were performed in 59 patients making the total number of dissections 415 . The following types of dissections were performed: inguinal (13\%), iliac (5\%), ilioinguinal (35\%), axillary $(20 \%)$ and neck (28\%).

\section{Characteristics}

Patient, tumor and lymph node characteristics are summarized in Table 1. Age characteristics were very similar for MUP (median 56, interquartile range [IQR] 44-66 years) and MKP (median 56, IQR 46-68 years) patients $(P=0.75)$. Gender was not significantly different between the MKP group (50\% male) and MUP group (57\% male) $(P=0.38)$. The site and the extent of the LND performed was not significantly different between both groups (all $P>0.05$ ). Adjuvant radiotherapy was given in $17.6 \%$ of MKP patients and $29.8 \%$ of MUP patients $(P=0.07)$. The mean and median follow-up for the entire population was 36 and 19 months, respectively (IQR 9-43). The mean and median follow-up for MUP was 40 and 24 months (IQR 14-49) against 35 and 19 months for MKP (IQR 8-42), respectively.

\section{Complications}

Of all patients, $44.4 \%$ experienced at least one complication during follow-up. Most frequent complications were wound infection and/or skin necrosis (17.8\%), seroma (16.9\%) and chronic lymph edema (12.3\%). For patients who underwent an inguinal LND, 59.1\% experienced at least one complication, while at least one complication was found in $37.5 \%$ of patients who underwent an iliac LND, in $65.0 \%$ of patients who underwent an ilioinguinal LND, in $26.5 \%$ of patients who underwent an axillary dissection and in $25.5 \%$ of patients who underwent a neck dissection. Chronic lymph edema was present in $11.4 \%$ of patients who underwent an inguinal LND, in $31.3 \%$ of patients who underwent an iliac LND, in $24.2 \%$ of patients who underwent an ilioinguinal LND and in $1.5 \%$ and $2.1 \%$ of patients who underwent an axillary and neck LND, respectively. MUP patients had at least one complication in $55.3 \%$ and chronic lymph edema was present in $12.5 \%$.
TABLE 1 Baseline characteristics of patient, primary site, and metastatic melanoma for patients with a known primary site (MKP, $n=295$ ) and patients with an unknown primary site (MUP, $n=47$ )

\begin{tabular}{|c|c|c|c|c|c|}
\hline \multirow[t]{2}{*}{ Characteristic } & \multicolumn{2}{|c|}{ MKP } & \multicolumn{2}{|c|}{ MUP } & \multirow[t]{2}{*}{$P$-value* } \\
\hline & $N$ & $\%$ & $N$ & $\%$ & \\
\hline \multicolumn{6}{|l|}{ Gender } \\
\hline Male & 149 & 50.5 & 27 & 57.4 & \\
\hline Female & 146 & 49.5 & 20 & 42.6 & 0.38 \\
\hline \multicolumn{6}{|l|}{ Age } \\
\hline Median (IQR) & \multicolumn{2}{|c|}{$56(44-66)$} & \multicolumn{2}{|c|}{$56(46-68)$} & 0.75 \\
\hline \multicolumn{6}{|l|}{ Site of primary } \\
\hline Head/neck & 61 & 20.7 & - & - & \\
\hline Trunk & 88 & 29.8 & - & - & \\
\hline Extremity & 141 & 47.8 & - & - & \\
\hline Other & 5 & 1.7 & - & - & NA \\
\hline \multicolumn{6}{|l|}{ Breslow thickness } \\
\hline $\mathrm{T} 1$ & 35 & 11.9 & - & - & \\
\hline $\mathrm{T} 2$ & 80 & 27.1 & - & - & \\
\hline T3 & 72 & 24.4 & - & - & \\
\hline $\mathrm{T} 4$ & 75 & 25.4 & - & - & \\
\hline Missing & 33 & 11.2 & - & - & NA \\
\hline \multicolumn{6}{|l|}{ Histology } \\
\hline NM & 89 & 30.2 & - & - & \\
\hline SSM & 64 & 21.7 & - & - & \\
\hline Other & 17 & 5.8 & - & - & \\
\hline Missing & 125 & 42.4 & - & - & NA \\
\hline \multicolumn{6}{|l|}{ Cark level } \\
\hline II & 13 & 4.4 & - & - & \\
\hline III & 69 & 23.4 & - & - & \\
\hline IV & 115 & 39.0 & - & - & \\
\hline V & 34 & 11.5 & - & - & \\
\hline Missing & 64 & 21.7 & - & - & NA \\
\hline \multicolumn{6}{|l|}{ Ulceration } \\
\hline Absent & 217 & 73.6 & - & - & \\
\hline Present & 78 & 26.4 & - & - & NA \\
\hline \multicolumn{6}{|l|}{ Site of TLND } \\
\hline Inguinal & 39 & 13.2 & 5 & 10.6 & \\
\hline Iliac & 15 & 5.1 & 1 & 2.1 & \\
\hline Ilioinguinal & 103 & 34.9 & 17 & 36.2 & \\
\hline Axillary & 58 & 19.7 & 10 & 21.3 & \\
\hline Neck & 80 & 27.1 & 14 & 29.8 & 0.89 \\
\hline \multicolumn{6}{|c|}{ Nr. of harvested nodes } \\
\hline Median (IQR) & $16(1$ & & 17 & 28) & 0.55 \\
\hline \multicolumn{6}{|c|}{ Nr. of positive nodes } \\
\hline Median (IQR) & $2(1-$ & & 1( & & 0.71 \\
\hline \multicolumn{6}{|l|}{ AJCC staging } \\
\hline N1 & 124 & 42.0 & 24 & 51.1 & \\
\hline N2 & 86 & 29.2 & 6 & 12.8 & \\
\hline N3 & 70 & 23.7 & 14 & 29.8 & \\
\hline Missing & 15 & 5.8 & 3 & 6.4 & 0.07 \\
\hline
\end{tabular}


TABLE 1 continued

\begin{tabular}{lllll}
\hline Characteristic & MKP & & MUP & $P$-value* \\
\cline { 2 - 2 } & $N$ & $N \quad \%$ &
\end{tabular}

$\mathrm{LN}$ ratio $(\%)$

Median (IQR) $11.6(6.3-26.3) \quad 8.2(4.8-32.3) \quad 0.38$

ECE

\begin{tabular}{lccccc} 
No & 205 & 69.5 & 29 & 61.7 & \\
Yes & 90 & 31.5 & 18 & 38.3 & 0.31 \\
\multicolumn{2}{l}{ Adjuvant radiotherapy } & & & & \\
No & 243 & 82.4 & 33 & 70.2 & \\
Yes & 52 & 17.6 & 14 & 29.8 & 0.07
\end{tabular}

Nr. of TLND performed

$\begin{array}{llllll}1 & 295 & 81.7 & 47 & 87.0 & \\ >1 & 66 & 18.3 & 7 & 13.0 & 0.88\end{array}$

$N M$ nodular melanoma, SSM superficial spreading melanoma, $L N$ lymph node, $N A$ not applicable

* $P$ values were calculated by Fisher exact test, chi-square test, or Mann-Whitney $U$-test

MKP patients had at least one complication in $55.6 \%$ and chronic lymph edema was present in $12.8 \%$.

The median duration of hospitalization was 5 days (IQR 3-8 days) for both MUP and MKP patients $(P=0.484)$.

\section{Survival}

Univariate analyses demonstrated that the following factors statistically significantly affected DFS: site of dissection, number of positive nodes, node positive ratio, ECE (Table 2). Multivariate analyses for DFS showed three significant prognostic factors: N2 $(P=0.04), \mathrm{N} 3(P<0.001)$ and ECE $(P=0.004)$ (Table 3). Gender, age, the number of collected lymph nodes, primary site, Clark level, histology of the primary, MUP and adjuvant radiotherapy were not statistically significant. Hazard ratios and $P$-values of all analyzed factors are summarized in Tables 2 and 3.

As for OS, ulceration, site of dissection, node positive ratio, number of positive nodes, ECE and MUP were statistically significant prognostic factors (Table 2). Ulceration was not known for MUP patients and not included in multivariate analyses. Multivariate analysis for OS revealed two highly significant factors associated with worse prognosis: the presence of ECE and N3 status (both $P<0.001)$. Two factors were associated with significant better prognosis: MUP $(P=0.03)$ and neck dissections $(P=0.04)$ (Table 3).

The estimated 5-year DFS rates for ECE were an estimated 12\% when present and $26 \%$ when absent $(P<0.001)$. For nodal status, 5-year DFS rates were $31 \%$, $17 \%$ and $9 \%$ for the respective N1, N2 and N3 categories
TABLE 2 Univariate analyses of prognostic factors for DFS and OS

\begin{tabular}{|c|c|c|c|c|c|c|}
\hline \multirow[t]{2}{*}{ Variable } & \multicolumn{3}{|l|}{ DFS } & \multicolumn{3}{|l|}{ OS } \\
\hline & HR & $95 \% \mathrm{CI}$ & $P$-value & HR & $95 \% \mathrm{CI}$ & $P$-valu \\
\hline \multicolumn{7}{|l|}{ Gender } \\
\hline Female & 1 & & & 1 & & \\
\hline Male & 1.03 & $0.80-1.33$ & 0.81 & 1.22 & $0.94-1.59$ & 0.13 \\
\hline \multicolumn{7}{|l|}{ Age } \\
\hline$\leq 50$ years & 1 & & & 1 & & \\
\hline$>50$ years & 0.94 & $0.73-1.21$ & 0.63 & 1.01 & $0.77-1.32$ & 0.94 \\
\hline \multicolumn{7}{|c|}{ Site of primary lesion } \\
\hline Extremity & 1 & & & 1 & & \\
\hline Head/neck & 0.77 & $0.53-1.11$ & 0.16 & 0.81 & $0.55-1.18$ & 0.26 \\
\hline Trunk & 1.34 & $0.99-1.82$ & 0.06 & 1.30 & $0.95-1.78$ & 0.11 \\
\hline Other & 0.54 & $0.22-2.20$ & 0.54 & 0.49 & $0.11-1.97$ & 0.31 \\
\hline Unknown & 0.73 & $0.63-1.38$ & 0.73 & 0.70 & $0.45-1.08$ & 0.10 \\
\hline \multicolumn{7}{|l|}{ MUP } \\
\hline No & 1 & & & 1 & & \\
\hline Yes & 0.92 & $0.64-1.31$ & 0.63 & 0.68 & $0.45-1.03$ & 0.07 \\
\hline \multicolumn{7}{|l|}{ Breslow } \\
\hline $\mathrm{T} 1$ & 1 & & & 1 & & \\
\hline $\mathrm{T} 2$ & 1.37 & $0.85-2.21$ & 0.20 & 1.54 & $0.91-2.63$ & 0.11 \\
\hline $\mathrm{T} 3$ & 1.78 & $1.10-2.89$ & 0.02 & 1.80 & $1.05-3.07$ & 0.03 \\
\hline $\mathrm{T} 4$ & 1.18 & $0.72-1.94$ & 0.51 & 1.62 & $0.95-2.76$ & 0.08 \\
\hline MUP & 1.21 & $0.72-2.06$ & 0.47 & 1.08 & $0.59-1.97$ & 0.81 \\
\hline \multicolumn{7}{|l|}{ Histology } \\
\hline SSM & 1 & & & 1 & & \\
\hline NM & 1.07 & $0.74-1.55$ & 0.73 & 1.10 & $0.75-1.61$ & 0.64 \\
\hline MUP & 0.91 & $0.59-1.41$ & 0.68 & 0.71 & $0.44-1.15$ & 0.16 \\
\hline Other & 0.70 & $0.36-1.39$ & 0.31 & 0.94 & $0.49-1.82$ & 0.85 \\
\hline
\end{tabular}

Clark level

\begin{tabular}{|c|c|c|c|c|c|c|}
\hline II & 1 & & & 1 & & \\
\hline III & 0.88 & $0.46-1.69$ & 0.70 & 1.16 & $0.55-2.45$ & 0.70 \\
\hline IV & 0.96 & $0.51-1.80$ & 0.90 & 1.11 & $0.54-2.29$ & 0.78 \\
\hline V & 0.85 & $0.41-1.73$ & 0.64 & 1.12 & $0.51-2.51$ & 0.77 \\
\hline MUP & 0.83 & $0.42-1.64$ & 0.60 & 0.79 & $0.36-1.74$ & 0.55 \\
\hline \multicolumn{7}{|l|}{ Ulceration } \\
\hline Absent & 1 & & & 1 & & \\
\hline Present & 1.21 & $0.90-1.63$ & 0.2 & 1.48 & $1.10-1.99$ & 0.01 \\
\hline \multicolumn{7}{|l|}{ Site of TLND } \\
\hline Inguinal & 1 & & & 1 & & \\
\hline Iliac & 0.97 & $0.47-1.98$ & 0.93 & 0.96 & $0.49-1.87$ & 0.90 \\
\hline Ilioinguinal & 0.76 & $0.50-1.14$ & 0.18 & 0.71 & $0.47-1.07$ & 0.10 \\
\hline Axillary & 1.14 & $0.73-1.76$ & 0.57 & 1.06 & $0.68-1.67$ & 0.78 \\
\hline Neck & 0.63 & $0.41-0.97$ & 0.04 & 0.61 & $0.39-0.94$ & 0.03 \\
\hline $\begin{array}{l}\text { No. of } \\
\text { collected } \\
\text { nodes }\end{array}$ & 1.00 & $0.99-1.01$ & 0.54 & 1.00 & $0.99-1.01$ & 0.65 \\
\hline \multicolumn{7}{|l|}{ AJCC staging } \\
\hline N1 & 1 & & & 1 & & \\
\hline $\mathrm{N} 2$ & 1.34 & $0.98-2.50$ & 0.07 & 1.33 & $0.96-1.84$ & 0.09 \\
\hline
\end{tabular}


TABLE 2 continued

\begin{tabular}{|c|c|c|c|c|c|c|}
\hline \multirow[t]{2}{*}{ Variable } & \multicolumn{3}{|l|}{ DFS } & \multicolumn{3}{|l|}{ OS } \\
\hline & HR & $95 \%$ CI & $P$-value & HR & $95 \% \mathrm{CI}$ & $P$-value \\
\hline N3 & 1.90 & $1.39-2.58$ & $<0.001$ & 1.97 & $1.43-2.72$ & $<0.001$ \\
\hline $\mathrm{LN}$ ratio & 2.35 & $1.51-3.64$ & $<0.001$ & 2.83 & $1.80-4.45$ & $<0.001$ \\
\hline \multicolumn{7}{|l|}{ ECE } \\
\hline Absent & 1 & & & 1 & & \\
\hline Present & 1.60 & $1.23-2.08$ & $<0.001$ & 1.83 & $1.39-2.40$ & $<0.001$ \\
\hline \multicolumn{7}{|c|}{ Adjuvant radiotherapy } \\
\hline No & 1 & & & 1 & & \\
\hline Yes & 1.13 & $0.83-1.53$ & 0.45 & 1.18 & $0.85-1.63$ & 0.33 \\
\hline
\end{tabular}

$H R$ hazard ratio, $C I$ confidence interval, SSM superficial spreading melanoma, $N M$ nodular melanoma, $L N$ lymph node

TABLE 3 Multivariate analyses of prognostic factors in all patients $(n=342)$ for DFS and OS

\begin{tabular}{llrllc}
\hline Characteristic & Variable & \multicolumn{1}{l}{$N$} & HR & $95 \%$ CI & $P$ \\
\hline DFS & & & & & \\
AJCC staging & N1 & 148 & 1 & & \\
& N2 & 92 & 1.40 & $1.02-1.93$ & 0.04 \\
& N3 & 84 & 2.08 & $1.47-2.94$ & $<0.001$ \\
ECE & No & 234 & 1 & & \\
OS & Yes & 108 & 1.57 & $1.16-2.12$ & 0.004 \\
ECE & & & & & \\
MUP & No & 285 & 1 & & \\
AJCC staging & Nes & 130 & 1.69 & $1.25-2.28$ & 0.001 \\
& No & 47 & 1 & & \\
& Yes & 295 & 0.62 & $0.40-0.96$ & 0.03 \\
N2 & 148 & 1 & & \\
Dissection type & Inguinal & 44 & 1 & & 0.15 \\
& Axillary & 68 & 0.74 & $0.47-1.16$ & 0.19 \\
& Neck & 94 & 0.61 & $0.38-0.97$ & 0.04 \\
\hline
\end{tabular}

$H R$ hazard ratio, $C I$ confidence interval

$(P<0.001)$. The 5-year DFS rate for MKP patients was $21 \%$, while the 5-year DFS rate for MUP patients was $25 \%$ $(P=0.619)$.

Absence of ECE demonstrated a 5-year OS of 36\% compared to $15 \%$ when present $(P<0.001)$ (Fig. 1a). The estimated 5-year OS rates for the different categories of nodal status (N1, N2 and N3) were $36 \%, 30 \%$ and $16 \%$, respectively $(P<0.001)$ (Fig. 1b). The 5 -year estimated Kaplan-Meier OS rate for MUP versus MKP showed a trend toward a better survival for MUP at $43 \%$ vs. $27 \%$ for MKP, respectively $(P=0.06)$ (Fig. 1 C).

\section{DISCUSSION}

In this retrospective study, 342 melanoma patients treated with a total of 415 TLND for palpable lymph nodes metastases were analyzed. Outcome of melanoma patients with an unknown primary site (MUP) were compared with patients with a known site of the primary melanoma lesion (MKP). After multivariate analysis, a statistically significant OS benefit was found for patients with MUP over MKP patients. Five-year OS rates were $43 \%$ for MUP patients and $27 \%$ for MKP patients $(P=0.03)$.

Cormier et al. demonstrated a significant survival benefit for 71 MUP patients in a multivariate Cox proportional hazard model when adjusted for nodal status, dissection site, age, gender and adjuvant therapy as well $(P=0.006)$. The 5-year OS rates were $55 \%$ and $42 \%$ for MUP versus MKP, respectively, with a median follow-up of 92 months. ${ }^{5}$ Lee et al. demonstrated similar results with 5 -year OS rates of 55\% for MUP patients $(N=262)$ and $44 \%$ for MKP patients $(N=1309)$, with a median follow up of 36 months. Again MUP was identified as a significant prognostic factor in multivariate analysis $(P=0.0001) .{ }^{4}$ Where previous mentioned studies revealed a small increased 5-year OS rate compared to our results, Chang et al. reported similar 5-year OS rates for both groups; $46 \%$ for MUP and $49 \%$ for MKP. ${ }^{3}$

All the above mentioned studies demonstrated higher 5-year OS rates for MKP (42\%-49\%) compared to our results $(27 \%)$. No statistically significant differences were found in patient and tumor characteristics between all studies. ${ }^{3-5}$ A reason for the worse survival of MKP patients in the present study might be the short median follow-up. Moreover, a tertiary referral center might perform surgery in patients with more advanced cases which might lead to worse survival.

A possible hypothesis for the survival benefit seen in MUP patients is an endogenous immune response, which also might have caused regression of the primary lesion. Interleukin- 2 and IFN- $\alpha$ have shown some therapeutic benefit, supposedly by enhancing antitumor immune responses. ${ }^{10-12}$ In a small study Moschos et al. treated 20 patients with stage IIIB and IIIC disease with neoadjuvant high-dose IFN- $\alpha 2 b$. Three were diagnosed with MUP and demonstrated no evidence of disease after 7, 9 and 10 months, respectively. ${ }^{13}$ Furthermore, cytoreductive surgery (complete metastasectomy) revealed a long-term clinical benefit that depended on the host's immune response to a surgical reduction in tumor burden. ${ }^{14}$ Causes for the effectiveness of these therapies in MUP patients might be the favorable patients' immune system. Unfortunately, no specific data are available to prove a difference between survival of MUP and MKP patients receiving any form of immunotherapy. 
FIG. 1 OS for (a) ECE, (b) number of positive lymph nodes according to AJCC staging system, and (c) MUP and MKP

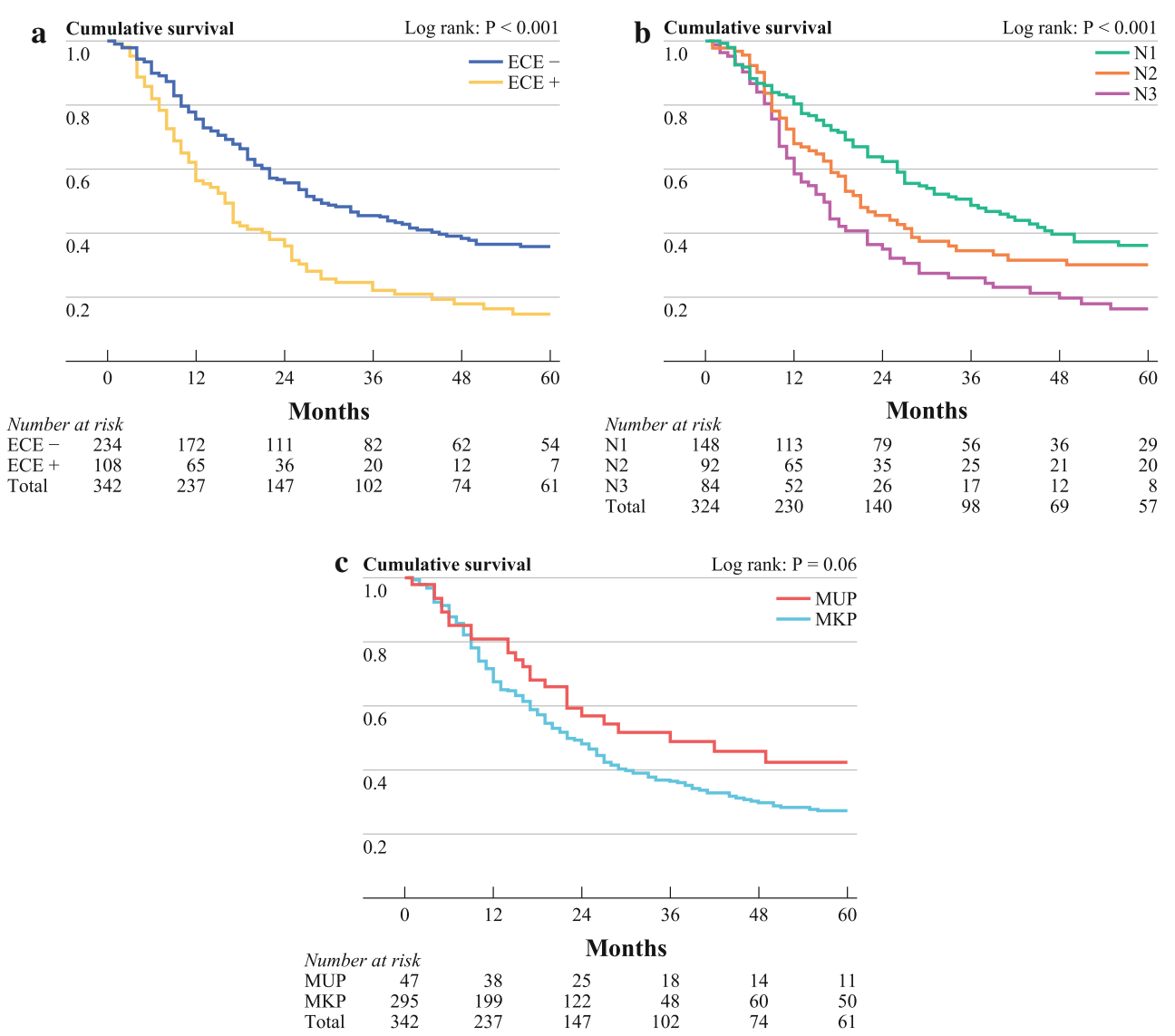

The rate of MUP in patients treated with TLND for palpable nodal disease was $13.7 \%$ in our institute (47 of 342 ). This is in line with other studies. In the study performed by Rutkowski et al., the rate was $12.8 \% .{ }^{15}$ Lee et al. demonstrated a MUP rate of $16.7 \%$, while the study performed by Cormier et al. had a MUP rate of $13.2 \%$.,5

Lymph node status and ECE are important statistically significant prognostic factors for OS, which has been demonstrated in several studies. ${ }^{4,15-18}$ A previous study from our group demonstrated that ECE was the most important prognostic factor for OS after TLND. ${ }^{16}$ A recent MUP study by Rutkowski et al also revealed ECE and lymph node status as significant factors for OS. ${ }^{15}$ Balch et al. found several significant prognostic factors for stage III melanoma such as nodal micrometastases, number of tumor-containing lymph nodes, Breslow thickness, patient age, ulceration, site of the primary and primary mitotic rate. Unfortunately MUP and ECE were not analyzed in their study. ${ }^{17}$ Disease of patients with metastatic melanoma from an unknown primary site, arising in lymph nodes, skin, or subcutaneous tissues, was clarified to be categorized as stage III rather than stage IV. ${ }^{19}$

The fact that patients treated with neck dissections had a survival benefit versus inguinal dissections is counterintuitive because head/neck melanomas are associated with worse prognosis. ${ }^{2,17,20}$ It may be a chance finding as the minimal significant difference $(P=0.04)$ could be explained by the small sample size, the difference in age (median 54 vs. 62 years) and percentage of ECE (24.4\% vs. $37.1 \%)$.

Comparing MUP patients with MKP patients stratified by Breslow thickness as T1, T2, T3 and T4 showed increased hazard ratios for T2 (hazard ratio $=1.54$ ), T3 (hazard ratio $=1.80)$ and $\mathrm{T} 4$ (hazard ratio $=1.62)$ versus a T1 tumor. MUP patients showed nearly the same hazard ratio (hazard ratio $=1.08$ ) as patients with $\mathrm{T} 1$ tumors at univariate analysis. Increasing Clark level was of little significance for OS, as did the MUP patients with unknown Clark scores. Patients with ulcerated primaries had significant worse OS $(P=0.01)$ compared to the unknown group. Also MUP patients have a survival benefit compared to patients with a primary tumor located on an extremity or trunk. It was previously suggested that MUP patients had a worse prognosis compared to patients with known primary tumors. ${ }^{3}$ These data suggest at least that MUP is not a significant negative prognostic factor compared to some stage III melanoma patients with known primary tumor characteristics.

A recent study in the Netherlands by Koomen et al. demonstrated that the incidence of noncutaneous melanoma 
is very rare. ${ }^{21}$ Therefore, the value of nasopharyngeal examination is doubtful and could be ignored when a patient presents with a palpable lymph node without a known primary tumor. A thorough physical examination in order to locate a primary tumor is still recommended. Also close examination of the skin of the drainage area of the metastatic lymph node could be considered in order to identify a regressive primary lesion. ${ }^{22}$

In conclusion, this study showed the presence of ECE, an increased number of positive nodes and patients with MKP as statistically significantly negative prognostic factors for OS. Patients with melanoma of an unknown primary site showed a statistically significantly better OS after multivariate analysis compared to patients with melanoma metastases from known primary tumors. Melanoma patients with palpable nodal disease and a MUP should be classified as stage III disease. The absence of a primary melanoma in stage III melanoma patients does not preclude surgery.

CONFLICT OF INTEREST None of the coauthors has a conflict of interest to declare.

OPEN ACCESS This article is distributed under the terms of the Creative Commons Attribution Noncommercial License which permits any noncommercial use, distribution, and reproduction in any medium, provided the original author(s) and source are credited.

\section{REFERENCES}

1. Rigel DS, Russak J, Friedman R. The evolution of melanoma diagnosis: 25 years beyond the ABCDs. CA Cancer J Clin. 2010; 60:301-16.

2. Balch CM, Soong SJ, Gershenwald JE, et al. Prognostic factors analysis of 17,600 melanoma patients: validation of the American Joint Committee on Cancer melanoma staging system. J Clin Oncol. 2001;19:3622-34.

3. Chang AE, Karnell LH, Menck HR. The National Cancer Data Base report on cutaneous and noncutaneous melanoma: a summary of 84,836 cases from the past decade. The American College of Surgeons Commission on Cancer and the American Cancer Society. Cancer. 1998;83:1664-78.

4. Lee CC, Faries MB, Wanek LA, Morton DL. Improved survival after lymphadenectomy for nodal metastasis from an unknown primary melanoma. J Clin Oncol. 2008;26:535-41.

5. Cormier JN, Xing Y, Feng L, et al. Metastatic melanoma to lymph nodes in patients with unknown primary sites. Cancer. 2006;106:2012-20.

6. Katz KA, Jonasch E, Hodi FS, et al. Melanoma of unknown primary: experience at Massachusetts General Hospital and Dana-Farber Cancer Institute. Melanoma Res. 2005;15:77-82.

7. Anbari KK, Schuchter LM, Bucky LP, et al. Melanoma of unknown primary site: presentation, treatment, and prognosis-a single institution study. University of Pennsylvania Pigmented Lesion Study Group. Cancer. 1997;79:1816-21.

8. Keilholz U, Punt CJ, Gore M, et al. Dacarbazine, cisplatin, and interferon-alfa-2b with or without interleukin-2 in metastatic melanoma: a randomized phase III trial (18951) of the European Organisation for Research and Treatment of Cancer Melanoma Group. J Clin Oncol. 2005;23:6747-55.

9. Eggermont AM, Suciu S, MacKie R, et al. Post-surgery adjuvant therapy with intermediate doses of interferon alfa $2 \mathrm{~b}$ versus observation in patients with stage IIb/III melanoma (EORTC 18952): randomised controlled trial. Lancet. 2005;366(9492): 1189-96.

10. Eggermont AM, Suciu S, Santinami M, et al. Adjuvant therapy with pegylated interferon alfa- $2 b$ versus observation alone in resected stage III melanoma: final results of EORTC 18991, a randomised phase III trial. Lancet. 2008;372(9633):117-26.

11. Ramirez-Montagut T, Turk MJ, Wolchok JD, et al. Immunity to melanoma: unraveling the relation of tumor immunity and autoimmunity. Oncogene. 2003;22:3180-7.

12. Konjevic G, Mirjacic Martinovic K, Vuletic A, Babovic N. Invitro IL-2 or IFN-alpha-induced NKG2D and CD161 NK cell receptor expression indicates novel aspects of NK cell activation in metastatic melanoma patients. Melanoma Res. 2010;20: 459-67.

13. Moschos SJ, Edington HD, Land SR, et al. Neoadjuvant treatment of regional stage IIIB melanoma with high-dose interferon alfa- $2 b$ induces objective tumor regression in association with modulation of tumor infiltrating host cellular immune responses. J Clin Oncol. 2006;24:3164-71.

14. Morton DL, Ollila DW, Hsueh EC, et al. Cytoreductive surgery and adjuvant immunotherapy: a new management paradigm for metastatic melanoma. CA Cancer J Clin. 1999;49:101-16, 65.

15. Rutkowski P, Nowecki ZI, Dziewirski W, et al. Melanoma without a detectable primary site with metastases to lymph nodes. Dermatol Surg. 2010;36:868-76.

16. van Akkooi AC, Bouwhuis MG, van Geel AN, et al. Morbidity and prognosis after therapeutic lymph node dissections for malignant melanoma. Eur J Surg Oncol. 2007;33:102-8.

17. Balch CM, Gershenwald JE, Soong SJ, et al. Multivariate analysis of prognostic factors among 2,313 patients with stage III melanoma: comparison of nodal micrometastases versus macrometastases. J Clin Oncol. 2010;28:2452-9.

18. Hughes TM, A'Hern RP, Thomas JM. Prognosis and surgical management of patients with palpable inguinal lymph node metastases from melanoma. Br J Surg. 2000;87:892-901.

19. Gershenwald JE, Soong SJ, Balch CM. 2010 TNM staging system for cutaneous melanoma....and beyond. Ann Surg Oncol. 2010;17:1475-7.

20. Grunhagen DJ, Eggermont AM, van Geel AN, et al. Prognostic factors after cervical lymph node dissection for cutaneous melanoma metastases. Melanoma Res. 2005;15:179-84.

21. Koomen ER, de Vries E, van Kempen LC, et al. Epidemiology of extracutaneous melanoma in the Netherlands. Cancer Epidemiol Biomarkers Prev. 2010;19:1453-9.

22. Bories N, Dalle S, Debarbieux S, et al. Dermoscopy of fully regressive cutaneous melanoma. Br J Dermatol. 2008;158: 1224-9. 